\title{
Ultrasonication of Bismuth Telluride Nanocrystals Fabricated by Solvothermal Method
}

\author{
Sang-Hyon Chu*a, Sang H. Choi ${ }^{\mathrm{b}}$, Jae-Woo Kim ${ }^{\mathrm{c}}$, Glen C. King ${ }^{\mathrm{b}}$ and James R. Elliott ${ }^{\mathrm{b}}$ \\ ${ }^{a}$ National Institute of Aerospace, Hampton, VA USA 23666; \\ bNASA Langley Research Center, Hampton, VA USA 23681; \\ ${ }^{\mathrm{c}}$ Science and Technology Corp., Hampton, VA USA 23666
}

\begin{abstract}
The objective of this study is to evaluate the effect of ultrasonication on bismuth telluride nanocrystals prepared by solvothermal method. In this study, a low dimensional nanocrystal of bismuth telluride $\left(\mathrm{Bi}_{2} \mathrm{Te}_{3}\right)$ was synthesized by a solvothermal process in an autoclave at $180^{\circ} \mathrm{C}$ and 200 psi. During the solvothermal reaction, organic surfactants effectively prevented unwanted aggregation of nanocrystals in a selected solvent while controlling the shape of the nanocrystal. The atomic ratio of bismuth and tellurium was determined by energy dispersive spectroscopy (EDS). The cavitational energy created by the ultrasonic probe was varied by the ultrasonication process time, while power amplitude remained constant. The nanocrystal size and its size distribution were measured by field emission scanning electron microscopy (FESEM) and a dynamic light scattering system. When the ultrasonication time increased, the average size of bismuth telluride nanocrystal gradually increased due to the direct collision of nanocrystals. The polydispersity of the nanocrystals showed a minimum when the ultrasonication was applied for 5 $\min$.
\end{abstract}

Keywords: bismuth telluride, nanocrystal, low-dimensional, ultrasonication, solvothermal

\section{INTRODUCTION}

Ultrasonication and nanoparticles

Nanoparticles have been of great interest since their large surface-to-volume ratio produces a dramatic enhancement of various material properties. In order to gain such benefits, it is of paramount importance that the size, shape and configuration of target nanoparticles be controlled. Usually nanoparticles are mixed with organic or inorganic matrix materials and homogeneous miscibility is another challenge in this case. The high surface-to-volume ratio of nanoparticle system gives rise to large surface interaction between nanoparticles, leading to aggregation of nanoparticles. For this reason, it is an extremely difficult challenge to obtain nanoparticles of uniform size while maintaining size homogeneity when mixed with other materials in the next processing stage.

There exist numerous methods of nanoparticle dispersion, such as chemical treatment of the particle surface, addition of surfactants, using a variety of dry powder processing devices, and using supercritical fluids as solvents [1]. In this study, we employed high-intensity ultrasonic waves to obtain nanometer-sized semiconductor particles. When the nanoparticles are dispersed in a liquid medium, the acoustic waves generated by an ultrasonication processor could be very effective in terms of particle dispersion by producing important non-linear effects in liquid media. The most dominant effects are transient cavitation and acoustic streaming [2]. They are responsible for homogeneous dispersion, refining the shape and structure of the individual particle, changing the surface morphology, and degassing of liquid medium. Under cyclic ultrasonic waves of high intensity, acoustic cavitation gives rise to the formation, growth, and collapsing of micro-bubbles in liquid media. The cavitation cycles of expanding and collapsing of micro-bubbles will involve micro "hot spots" where nanoparticles transiently experience extreme conditions: high temperatures of $\sim 5000 \mathrm{~K}$, pressures of about $1800 \mathrm{~atm}$, and heating and cooling rates above $10^{10} \mathrm{~K} / \mathrm{s}[3-8]$. Thus, strong impact generated by the ultrasonic cavitation can break up the aggregated nanoparticles and disperse them more uniformly in liquids. On the other hand, the high-intensity transient cavitations coupled with locally high temperature in a very short time could launch shock waves in solid- 
liquid systems. When nanoparticles in close proximity to one another receive these shock waves, high energy collision among nanoparticles can occur, inducing effective melting at the point of collision [5-8] and increased average particle size. In this regard, the control of the ultrasonication condition can allow for a variety of applications depending on the requirements and the final products. In this study, we focus on the preparation of uniform dispersion of nanoparticles/nanocrystals and the ultrasonication process to obtain the refined structure and dimension of nanoparticles/nanocrystals.

\section{Bismuth Telluride Nanocrystals (NCs) for Thermoelectrical (TE) applications}

Thermoelectric (TE) materials have been studied extensively due to their attractive applications in solid-state cooling and electric power generation. Back in 1957, it was already suggested TE semiconductors will replace moving parts or fluids in conventional cooling devices (ex. chloroflurocarbons (CFCs) or hydrochlorocarbons (HCFCs), which are harmful to environment) [9]. Presently, TE materials are being used for many other applications such as the solid state cooling of laser diodes, thermochemistry-on-a-chip, fiber-optic, microelectrothermal systems, and so forth [10-12].

The current use of TE devices is limited by relatively poor efficiencies. The performance of thermoelectric devices depends on the figure of merit (ZT) of the material, given by

$$
\mathrm{ZT}=\mathrm{S}^{2} \mathrm{~T} / \rho \kappa
$$

where S, T, $\rho$ and $\kappa$ are the Seebeck coefficient, the absolute temperature, the electrical resistivity and the thermal conductivity, respectively. High performance TE materials require an ideal combination of the electrical and thermal properties. The challenge lies in achieving simultaneously high power factor $\left(\mathrm{S}^{2} / \rho\right)$ and low thermal conductivity $(\kappa)$. Thermal conductivity is composed of the sum of the contributions from the lattice thermal conductivity (phonon) $\kappa_{\mathrm{L}}$, and the electronic thermal conductivity $\kappa_{\mathrm{e}}$. The Seebeck coefficient, the electrical conductivity, and the thermal conductivity are not independent, since they are determined by the details of the electronic structure and scattering of charge carriers. The increased electrical conductivity usually causes the thermal conductivity to increase at the same time. In the case of semiconductors, $\kappa_{\mathrm{L}}$ becomes dominant in the total thermal conductivity. Thus, much effort has been made to decrease the thermal conductivity. Recently, it was suggested that the concept of "phononglass/electron-crystal" (PGEC) model, such as skutterudites and clathrates, can lower the thermal conductivity by the strong phonon scattering [13-15]. Likewise, low-dimensional materials, such as quantum wells, superlattices, quantum wires, and quantum dots are another type of PGEC materials that offer innovative ways to manipulate the electron and phonon properties [16-18]. Some experimental results [19] have already shown that quantum size effects in low-dimensional materials with nanostructural characteristics can improve the TE device efficiency as predicted by the theoretical calculations. Bismuth telluride and its alloys are currently best-known materials for TE cooling devices due to their highest TE performance for operation at room temperature [20]. The attempts to fabricate low-dimensional bismuth telluride have been made in various ways such as chemical alloying method by coprecipitation [21], reverse micelle method [22], hydrothermal [23, 24] and solvothermal method [25, 26], elimination reaction method [27], organometallic method [28], electrochemical deposition [29, 30] etc. Shape and size tailoring becomes very critical since the overall TE figure of merit can be determined by the nanostructure of low-dimensional materials. The previous cases [21-30] have indicated that the shape and size control is not easy to achieve. In this study, bismuth telluride nanocrystals are fabricated via solvothermal method and their size and shape are tailored by surfactants during solvothermal reactions and ultrasonication in the post-process.

\section{EXPERIMENTAL}

All chemicals were purchased as analytical grade and used without further purification. In a typical synthesis process, bismuth chloride $\left(\mathrm{BiCl}_{3},>99 \%\right)$ and tellurium (Te, 99.999\%) powder were prepared into 2:3 molar ratio solution in N,N-dimethylformamide (DMF) solvent. Potassium hydroxide $(\mathrm{KOH})$ was added, and then organic surfactants [(ethylenediaminetetraacetic acid disodium salt (EDTA), cetyltrimethyl ammonium bromide (CTAB) or sodium dodecylsulfate (SDS)] were put into the bismuth telluride solution before a complete mixing. The final solution was transferred into a teflon-lined autoclave which was sealed immediately and heated to the reaction temperature of $180{ }^{\circ} \mathrm{C}$ (see Figure 1 for the autoclave setup). During the reaction, the solution was stirred by a stainless steel stirrer with a rotational speed of $100 \mathrm{rpm}$. After a reaction time of $24 \mathrm{hrs}$ at $180{ }^{\circ} \mathrm{C}$, the autoclave was 
naturally cooled down to room temperature. The precipitated dark grey products were filtered, washed with distilled water and absolute ethanol several times, and dried in vacuum at $80{ }^{\circ} \mathrm{C}$ for $24 \mathrm{hrs}$.

For the ultrasonication process, distilled water was added to the nanocrystal powder. The aqueous solutions of the bismuth telluride nanocrystals were put into borosilicate vials. The concentration of the aqueous sample was $>0.1 \mathrm{mg} / \mathrm{L}$ to ensure data reproducibility. The sealed vials were placed into the cup horn (probe) installed into ultrasonication generator $(20 \mathrm{kHz}$ and $600 \mathrm{~W})$ and transducer (converter), as shown in Figure 2. The cup horn setup prevented any contamination that could take place when the ultrasonication probe makes direct contact with the sample. The ultrasonication was operated below the cut-off temperature of $80{ }^{\circ} \mathrm{C}$ to minimize not only nanocrystal aggregation, but also evaporation of transmitter fluids which filled the cup horn. The pulse mode operation was selected for the same reasons. The variable conditions allowed here for the ultrasonication process were (1) ultrasonication amplitude (power) and (2) process time. The processing time was tested in the range of $0,1,5$, 10, 30 min while the amplitude was set constant in the current study.

The structural characterization of bismuth telluride nanocrystals was performed using high-resolution field emission scanning electron microscopy (FESEM, Hitachi S-5200). All FESEM images were obtained without any metal coating on the surface of the nanocrystals in order to eliminate any possible artifacts attributable to the coating layer. Simultaneously, energy dispersive spectroscopy (EDS) installed in FESEM system enabled determination of the atomic ratio of bismuth and tellurium. When various conditions of ultrasonication process were applied, the size characteristics of the bismuth nanocrystals were respectively measured by the dynamic light scattering (4mW He-Ne, 633nm).

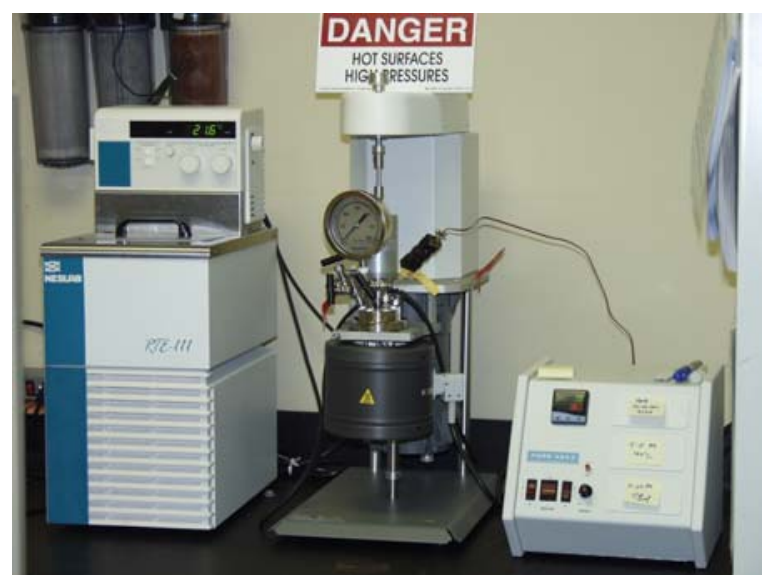

Figure. 1. Autoclave setup for solvothermal reaction of bismuth telluride nanocrystals.
Procedure of solvothermal synthesis of bismuth telluride nanocrystals
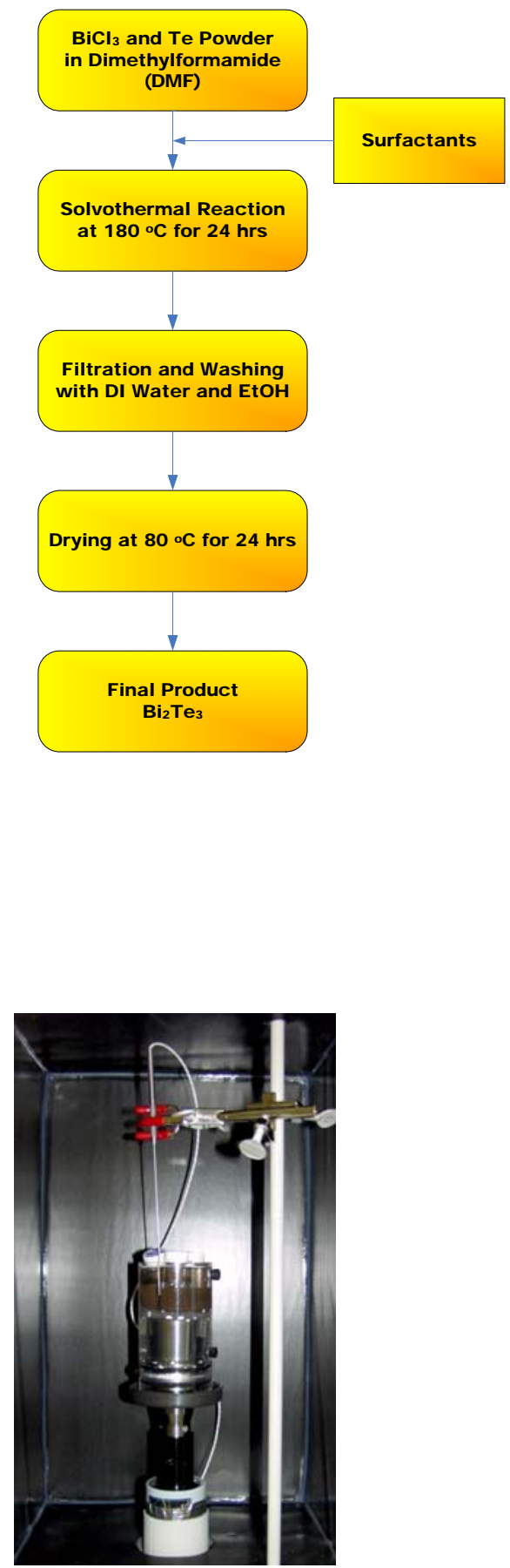

Figure. 2. Experimental setup of ultrasonication processor $(20 \mathrm{kHz}$ and $600 \mathrm{~W})$ with thermo-couple installed on behalf of temperature control. 


\section{RESULTS AND DISCUSSION}

\section{Synthesis of bismuth telluride nanocrystals}

The solvothermal synthesis successfully produced dark grey powder of bismuth telluride nanocrystal after separation procedure including the filtering, washing and drying, as described previously. A typical sample of bismuth telluride powder is shown in Figure 3(a). The production yield was around $95 \%$ on basis of bismuth telluride $\left(\mathrm{Bi}_{2} \mathrm{Te}_{3}, \mathrm{FW}=\right.$ $524.32 \mathrm{~g} / \mathrm{mol}$ ). Figure 3(b) and 3(c) exhibit FESEM images of bismuth telluride nanocrystals fabricated using $\mathrm{KOH}$ and EDTA. Especially, Figure 3(b) illustrates an example of low dimensional nanocrystal which can enhance TE properties. The size and shape of such nanocrystals of bismuth telluride can be controlled by selection of appropriate processing condition and type of surfactant and reducing agent. As shown here, the synthesized nanocrystals exist in the form of not only nanocrystals (nanosheets) but also their clusters. The break-up of the clusters was implemented using the ultrasonication process. The element analysis of bismuth telluride was measured by EDS analysis of FESEM (see Figure 3(d)). Table 1 gives the weight and atomic ratio of a typical bismuth telluride nanocrystal sample. The atomic ratio of $\mathrm{Bi}_{2.00} \mathrm{Te}_{2.95}$ confirmed that the solvothermal reaction successfully synthesized $\mathrm{Bi}_{2} \mathrm{Te}_{3}$.

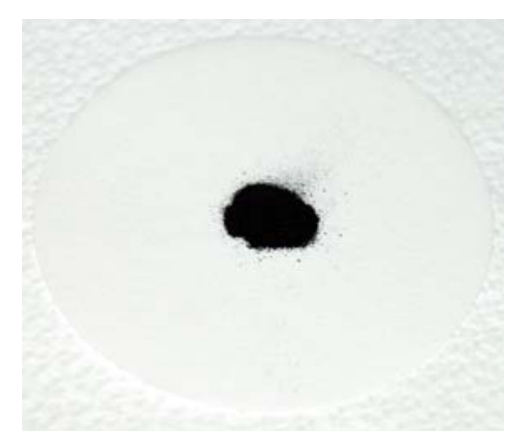

(a)

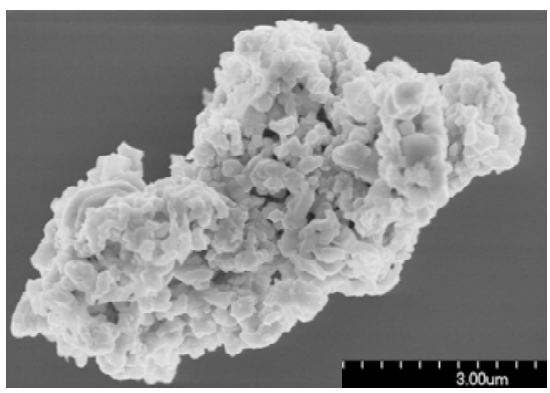

(c)

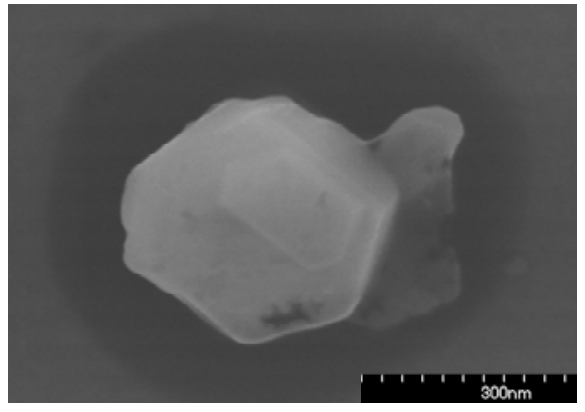

(b)

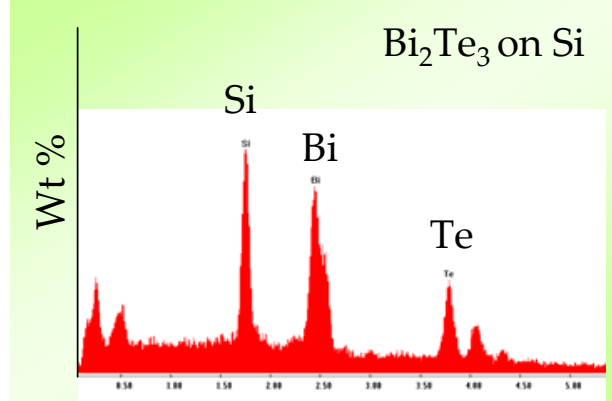

(d)

Figure 3. Images of nanocrystalline bismuth telluride: (a) bismuth telluride powder after solvothermal synthesis, (b) FESEM image of 2-dimensional nanosheets, (c) FESEM image of clustered nanocrystals, and (d) EDS element analysis data. 
Table 1. EDS Element Analysis of Bismuth Telluride Nanocrystals fabricated solvothermally using KOH and EDTA

\begin{tabular}{|c|c|c|}
\hline & Weight \% & Atomic \% \\
\hline $\operatorname{Bi}(\mathrm{M})$ & 52.64 & 40.43 \\
\hline $\mathrm{Te}(\mathrm{L})$ & 47.36 & 59.57 \\
\hline
\end{tabular}

The effect of ultrasonication on the nanocrystal morphology

The ultrasonication frequency $(20 \mathrm{kHz})$ and amplitude were constant and the ultrasonication process time was varied in the range of $0,1,5,10,30 \mathrm{~min}$, in order to investigate the effect on the nanocrystal morphology. The average diameter $(d)$ of the nanocrystal sample and the polydispersity index (PDI) of the nanocrystal size distribution were measured by a He-Ne laser. The average diameter is the Z-average diameter based on the intensity peak as a function of the nanocrystal size. (see Figure 4). The Z-average diameter, known as the cumulants mean, is an intensity mean because it is calculated from the signal intensity. Figure 4 shows each intensity peak obtained with different processing time given. All the size distribution data showed bimodal curves. Without any ultrasonication (0-min case), it was impossible to measure the dynamic light scattering signal because of too much noise in the scattering data. After irradiation of ultrasonic energy for $5 \mathrm{~min}$, the Z-average diameter of the nanocrystal was measured as $480 \mathrm{~nm}$. As the ultrasonication time increased, the nanocrystal size in the aqueous solution also increased up to $2.2 \mu \mathrm{m}$. Interestingly, a small peak between 5 and $6 \mu \mathrm{m}$ increased simultaneously when more ultrasonic irradiation was applied. In the case of 30-min sample, such particle aggregation effect became so severe that the size intensity curve showed dominant existence of large bismuth particles $(>1 \mu \mathrm{m})$. The intensity curve also became very broad. Clearly, the size analysis of the nanocrystal indicated the ultrasonic irradiation gave a strong impact to the nanocrystal size, welding the nanocrystals together by a direct collision between the smaller nanocrystals (see Figure 5). Besides, a collision of the nanocrystals at a glancing angle can result into a mechanical smoothing of the surface. The FESEM study is currently going on to investigate the smoothing effect of the nanocrystal surface.

Another parameter obtained from the cumulants analysis of the dynamic light scattering is polydispersity index (PDI) which represents a width of the size intensity curve. The Z-average diameter and the PDI of the nanocrystal are summarized as a function of the processing time in Figure 5. When compared with the size data, the PDI value of each bismuth telluride sample gave a very interesting result. The nanocrystal size distribution exhibited a minimum PDI of 0.26 in the case of the sample irradiated for $5 \mathrm{~min}$. This result implies that the ultrasonication is also beneficial in terms of narrowing the nanocrystal size distribution. Currently, more tests are underway to confirm such an interesting behavior.

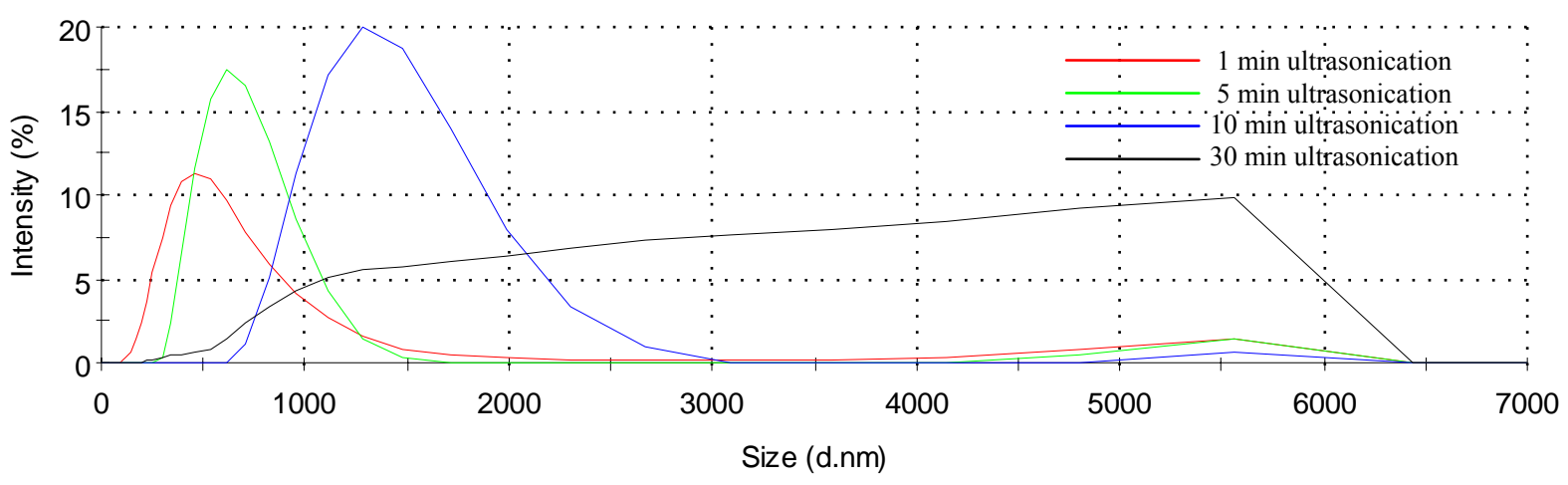

Figure 4. The size distribution curves of the bismuth telluride nanocrystals after the ultrasonication processes with different processing time. All data were measured by the dynamic light scattering system ( $4 \mathrm{~mW}$ He-Ne laser, $633 \mathrm{~nm})$ 


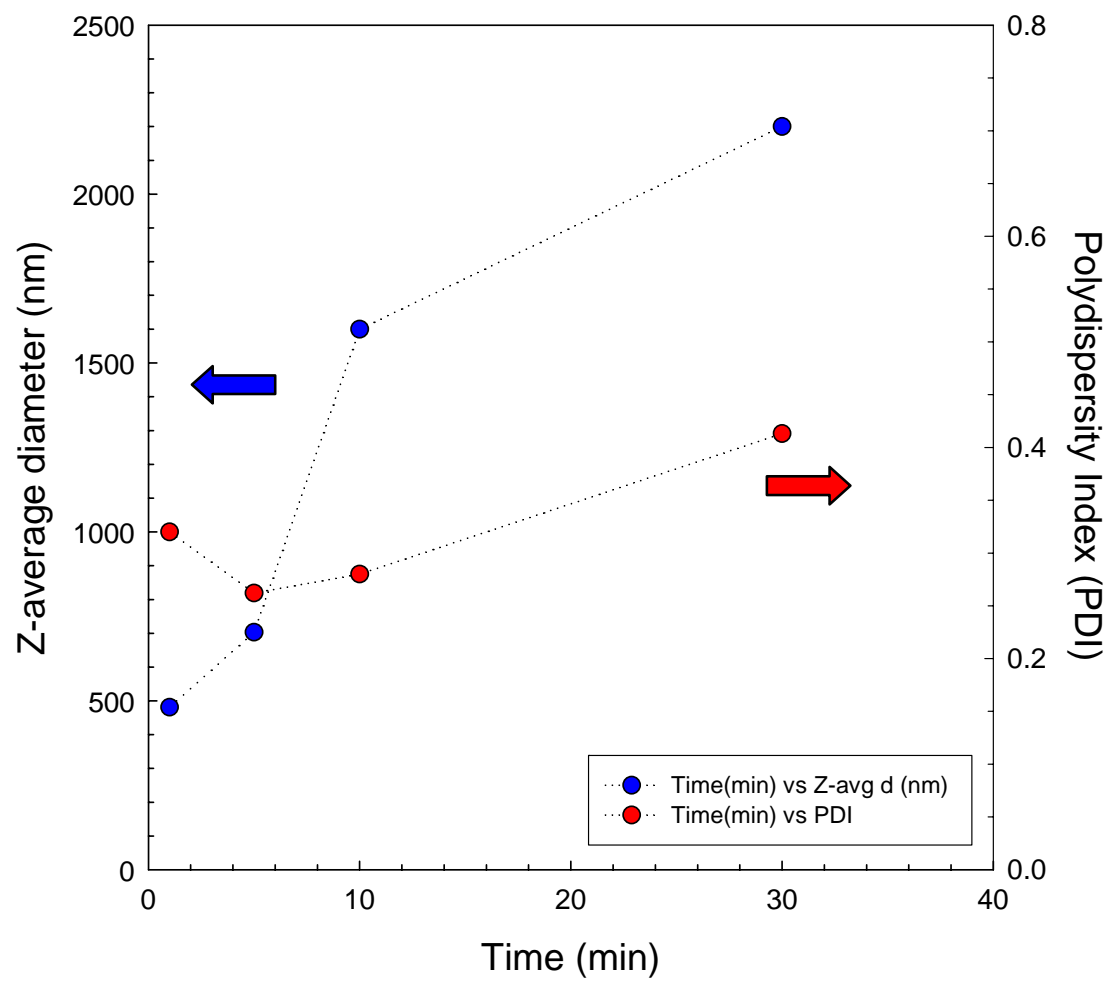

Figure 5. The effects of the ultrasonication on the Z-average size and polydispersity index of the bismuth telluride nanocrystal. All data were also measured by the dynamic light scattering system. The current ultrasonication condition produced the aggregation of the nanocrystal particles due to a direct collision between them while narrowing the polydispersity index to 0.26 for a processing time of $5 \mathrm{~min}$.

\section{SUMMARY}

Bismuth telluride is one of very promising thermoelectric materials at room temperature. In this study, solvothermal synthesis successfully produced low-dimensional nanocrystals of bismuth telluride. To study the effect of the ultrasonication on the dispersion of the low-dimensional nanocrystals, the ultrasonication time was varied and the bismuth telluride nanocrystal powder was characterized by the dynamic scattering technique. The result was summarized as follows:

- The effect of the ultrasonic wave was so large that the ultrasonication treatment dramatically changed the size and the size distribution of the bismuth telluride nanocrystals when the processing time was varied with a given frequency and amplitude of the ultrasonic wave.

- The average size of the semiconducting nanocrystal increased with the processing time increased. This result was attributed to a direct collision of the nanocrystal particle. The high-speed collision induced aggregation of the smaller nanocrystals and the increase of the Z-average diameter of the bismuth telluride nanocrystal. 
- The size distribution and polydispersity became narrower when the ultrasonic wave was applied to the aqueous solution of the bismuth telluride for $5 \mathrm{~min}$. As the processing time was increased to 10 and 30 $\mathrm{min}$, the polydispersity index increased, however.

\section{REFERENCES}

1. Y. Hakuta , H. Hayashi, and K. Arai, "Fine particle formation using supercritical fluids," Current Opinion in Solid State and Materials Science, 7(4-5), 341-351, 2003.

2. K. M. Mussert, W. P. Vellinga, A. Bakker, and S. Van Der Zwaag, "A nano-indentation study on the mechanical behaviour of the matrix material in an AA6061-A12O3 MMC," Journal of Materials Science, 37(4), 789-794, 2002.

3. K. S. Suslick and L. A. Crum, Encyclopedia of Acoustics, ed. M. J. Crocker, 1, New York, NY, WileyInterscience, 271-282, 1997.

4. K. S. Suslick and L. A. Crum, "APPLICATIONS OF ULTRASOUND TO MATERIALS CHEMISTRY," Annual Review of Materials Science, 29, 295-326, 1999.

5. K. S. Suslick, S. J. Doktycz, and E. B. Flint, "On the Origin of Sonoluminescence and Sonochemistry," Ultrasonics, 28 (5), 280-290, 1990.

6. S. J. Doktycz, K. S. Suslick, "Interparticle Collisions Driven by Ultrasound," 247, 1067-1069, 1990.

7. K. S. Suslick, and S. J. Doktycz, Advances in Sonochemistry, ed. T. J. Mason, 1:197-230. Greenwich, CT, JAI, 1990.

8. T. Prozorov, R. Prozorov, and K. S. Suslick, "High Velocity Inter-Particle Collisions Driven by Ultrasound," J. Am. Chem. Soc. 126, 13890-13891, 2004.

9. A. E. Ioffe, Semiconductor Thermoelements and Thermoelectric Cooling, Infosearch Limited, London, 1957.

10. D. M. Rowe, Handbook of Thermoelectroics, CRC Press, Boca Raton, FL, 595, 1995.

11. F. J. DiSalvo, “Thermoelectric Cooling and Power Generation," Science, 285, 30, 703-706, 1999.

12. R. Venkatasubramanian, E. Siivola, T. Colpitts, and B. O'Quinn, "Thin-Film Thermoelectric Devices with High Room-Temperature Figures of Merit," Nature, 413, 597-602, 2001.

13. G. A. Slack, CRC Handbook of Thermoelectrics, ed. by D. M. Rowe, CRC, Boca Raton, FL, 401, 1995.

14. G. S. Nolas, G. A. Slack, D. T. Morelli, T. M. Tritt, and A. C. Ehrlich, "The effect of rare-earth filling on the lattice thermal conductivity of skutterudites," Journal of Applied Physics, 79 (8), 4002-4008, 1996.

15. G. S. Nolas, J. L. Cohn, G. A. Slack, and S. B. Schujman, "Semiconducting Ge clathrates: Promising Candidates for Thermoelectric Applications," Applied Physics Letters, 73 (2), 178-180, 1998.

16. G. Chen, M. S. Dresselhaus, G. Dresselhaus, J.-P. Fleurial, and T. Caillat, "Recent Developments in Thermoelectric Materials," International Materials Reviews, 48(1), 45-64, 2003.

17. L. D. Hicks, M. S. Dresselhaus, "Effect of Quantum-Well Structures on the Thermoelectric Figure of Merit," Physical Review B, 47, 12727-12731, 1993.

18. L. D. Hicks, M. S. Dresselhaus, "Thermoelectric Figure of Merit of a One-dimensional Conductor," Physical Review B, 47, 16631-16634, 1993.

19. S.-H. Yu, M. Yoshimura, "Shape and Phase Control of ZnS Nanocrystals: Template Fabrication of Wurtzite $\mathrm{ZnS}$ Single-Crystal Nanosheets and $\mathrm{ZnO}$ Flake-like Dendrites from a Lamellar Molecular Precursor $\mathrm{ZnS} \cdot\left(\mathrm{NH}_{2} \mathrm{CH}_{2} \mathrm{CH}_{2} \mathrm{NH}_{2}\right)_{0.5}, "$ Advanced Materials, 14(4), 296-300, 2002.

20. T. M. Tritt, "THERMOELECTRIC MATERIALS: Holey and Unholey Semiconductors, Science, 283 (5403), $804-805,1999$.

21. M. Toprak, Y. Zhang, and M. Muhammed, "Chemical alloying and characterization of nanocrystalline bismuth telluride," Materials Letters, 4460, 1-7, 2003.

22. E. E. Foos, R. M. Stroud, and A. D. Berry, "Synthesis and Characterization of Nanocrystalline Bismuth Telluride," Nano Letters, 1(12), 693-695, 2001.

23. H. L. Ni, T. J. Zhu, and X. B. Zhao, Thermoelectric properties of hydrothermally synthesized and hot pressed ntype $\mathrm{Bi}_{2} \mathrm{Te}_{3}$ alloys with different contents of Te," Materials Science and Engineering B, 117, 119-122, 2005.

24. X. B. Zhao, X. H. Ji, Y. H. Zhang, T. J. Zhu, J. P. Tu, and X. B. Zhang, "Bismuth telluride nanotubes and the effects on the thermoelectric properties of nanotube-containing nanocomposites," Applied Physics Letters 86, $062111,2005$. 
25. Y. Deng, C.-W. Nan, G.-D. Wei, L. Guo, Y.-H. Lin, "Organic-Assisted Growth of Bismuth Telluride Nanocrystals," Chemical Physics Letters, 374, 410-415, 2003.

26. X. B. Zhao, X. H. Ji, Y. H. Zhang, B. H. Lu, "Effect of Solvent on the Microstructures of Nanostructured $\mathrm{Bi}_{2} \mathrm{Te}_{3}$ Prepared by Solvothermal Synthesis," Journal of Alloys and Compounds, 368, 349-352, 2004.

27. T. J. Groshens, R. W. Gedridge, C. Lowe-Ma, "Room-Temperature MOCVD of $\mathrm{Sb}_{2} \mathrm{Te}_{3}$ Films and Solution Precipitation of $\mathrm{M}_{2} \mathrm{Te}_{3}(\mathrm{M}=\mathrm{Sb}, \mathrm{Bi})$ Powders via a Novel (N,N-Dimethylamino)Trimethylsilane EliminationReaction," Chemistry of Materials, 6, 727-729, 1994.

28. J. J. Ritter and P. Maruthamuthu, "Synthesis of Polycrystalline Bismuth Telluride by a Metal-Organo Complex Method," Inorganic Chemistry, 34, 4278-4280, 1995.

29. S. A. Sapp, B. B. Lakshmi, C. R. Martin, "Template Synthesis of Bismuth Telluride Nanowires," Advanced Materials, 11, 402-404, 1999.

30. A. L. Prieto, M. S. Sander, M. S. Martín-González, R. Gronsky, T. Sands, and A. M. Stacy, "Electrodeposition of Ordered $\mathrm{Bi}_{2} \mathrm{Te}_{3}$ Nanowire Arrays," Journla of American Chemistry Society, 123, 7160-7161, 2001. 